\title{
Correction to: Microbiota and Aging
}

\author{
Maoyang Lu and Zhao Wang
}

\section{Correction to: \\ Chapter 9 in: Z. Wang (ed.) Aging and Aging-Related Diseases, Advances in Experimental Medicine and Biology 1086, https://doi.org/10.1007/978-981-13-1117-8_9}

The original version of the chapter was inadvertently published without an acknowledgement details. The acknowledgements section has now been updated with the below text:

The paragraph "Compared to evidence related to aging ... with an average age of 70 years" in Sect. 9.2 of this chapter was re-used from the original work "Buford, T.W. (Dis) Trust your gut: the gut microbiome in age-related inflammation, health, and disease. Microbiome 5, 80 (2017) doi:https://doi.org/10.1186/s40168-017-0296-0”. 